\title{
Abundances \& Abundance Ratios in our Galaxy \& the Local Group
}

\author{
Eline Tolstoy \\ Kapteyn Astronomical Institute, University of Groningen, Postbus 800, 9700AV Groningen, \\ the Netherlands
}

\begin{abstract}
This is a review of the progress of ESO/WFI Imaging and VLT/FLAMES spectroscopy of large numbers of individual stars in nearby dwarf spheroidal galaxies primarily by the Dwarf Abundances and Radial-velocities Team (DART). These observations have allowed us to show that neither the kinematics nor the abundance nor the spatial distributions are easy to explain in a straight forward manner for these smallest galaxies. The main result is that dwarf galaxies show complex and highly specific evolutionary and metal enrichment processes, especially at ancient times. This conclusively proves that these small galaxies are not the building blocks of the larger galaxies in the Local Group.
\end{abstract}

\section{Introduction}

It is likely that low mass dwarfs are the most common type of galaxy in the Universe, but because of their extremely low surface brightness our ability to detect them diminishes rapidly with increasing distance. The only place where we can be reasonably sure to detect a large fraction of these objects is in the Local Group, and even here, "complete samples" are added to each year (e.g., Belokurov et al. 2006). Only the closest galaxies can be observed in detail, even with an $8 \mathrm{~m}$ telescope.

The lowest mass galaxies are dwarf irregular (dI) and dwarf spheroidal (dSph) type galaxies. The only difference between these low mass dSphs and dIs seems to be the presence of gas and current star formation in dIs. It has already been noted (e.g., Einasto et al. 1974) that dSphs predominantly lie close to our galaxy ( $<250 \mathrm{kpc}$ away), and dIs predominantly further away ( $>400 \mathrm{kpc}$ away). This suggests that the proximity of $\mathrm{dSph}$ to our Galaxy played a role in the removal of gas from these systems. Although the range of properties found in $\mathrm{dSph}$ and $\mathrm{dI}$ galaxies does not allow a straight forward explanation; particularly not the large variations in star formation histories and chemical evolution paths that have now been observed in different systems (e.g., Dolphin et al. 2005).

\section{Observations}

VLT/FLAMES with fibre-feeds to the Giraffe and UVES spectrographs has made a revolution possible in spectroscopic studies of resolved stellar populations in nearby galaxies. The modes of operation, the sensitivity and the field of view are an almost perfect match to requirements for the study of Galactic dSph galaxies. For example, it is now possible to measure the abundance of numerous elements in nearby galaxies for more than 100 stars over a 25' diameter field of view in one shot (Hill et al. 2007, in prep; Letarte et al. 2007, in prep). A vast improvement on previous laborious (but valiant) efforts with single slit spectrographs to observe a handful of stars per galaxy (e.g., Tolstoy et al. 2003; Shetrone et al. 2003; Geisler et al. 2005).

The DART Large programme has measured abundances and velocities for several hundred individual likely member stars in a sample of four nearby dSph galaxies: Sculptor 
(513 members), Fornax (933 members), Sextans (202 members) and Carina (364 members). We have used the VLT/FLAMES facility in the low resolution mode (LR 8, $\mathrm{R} \sim 8000$ ) to obtain Ca II triplet metallicity estimates as well as accurate radial velocity measurements over a large areas out to the tidal radii (e.g., Tolstoy et al. 2004; Battaglia et al. 2006; Helmi et al. 2006), see Figure 1. Each of the galaxies have also been observed at high resolution (Giraffe settings HR 10,13 \&14) in the central region to obtain detailed abundances for a range of interesting elements such as $\mathrm{Mg}, \mathrm{Ca}, \mathrm{O}, \mathrm{Ti}, \mathrm{Na}, \mathrm{Eu}$ to name a few (Hill et al. 2007 in prep; Letarte et al. 2007 in prep; Babusiaux et al. 2007, in prep; Venn et al. 2007, in prep.) for about 90 stars per galaxy. During the Giraffe HR observations we were also able to use the fibre feed to the UVES spectrograph to obtain greater wavelength coverage and higher resolution for a sample of 7-14 stars per galaxy (e.g., Venn et al. 2007 in prep; Shetrone et al. 2007 in prep). The comparison we can make between results from UVES spectroscopy $(\mathrm{R} \sim 40000)$ and Giraffe spectroscopy ( $R \sim 20000)$ for the same stars is very useful in convincing ourselves, and others, that we are able to obtain reliable results with lower resolution spectra than was previously thought possible or advisable.

\section{Colour-Magnitude Diagrams}

The first step in a detailed analysis of the resolved stellar population of a galaxy is an accurate Colour-Magnitude Diagram, ideally down to the oldest main sequence turn-offs $\left(\mathrm{M}_{V} \sim+3.5\right)$. A careful analysis leads to the star formation history all the way back to the first star formation in the galaxy. This approach is the most accurate for intermediate age populations, but for stars older than about $10 \mathrm{Gyr}$ the time resolution gets quite poor (and the stars are getting very faint), and it becomes hard to distinguish a 12 Gyr old star from a 10 Gyr old star. Here it becomes useful to consider the Horizontal Branch stars $\left(\mathrm{M}_{V} \sim 0\right.$. $)$ which are the bright He-burning phase of low mass stars $>10$ Gyr old. The ratio of red to blue horizontal branch stars tells us about the age and metallicity variation (e.g., Lee et al. 2001).

We can also consider the much brighter Red Giant Branch (RGB, $-3<M_{V}<0$.) stars which have ages $>1$ Gyr old, back to the oldest stars in the galaxy, however the interpretation of the RGB using only photometry is plagued by the age-metallicity degeneracy (graphically illustrated by Cole et al. 2005; their Figure 8). The observed magnitude and colour (e.g., $\mathrm{M}_{V}, \mathrm{~V}-\mathrm{I}$ ) of a star combined with a measured $[\mathrm{Fe} / \mathrm{H}]$, allows us to effectively remove the age-metallicity degeneracy and determine the age of an RGB star from an isochrone, and thus to trace the enrichment patterns of as many elements as we can measure with time.

\section{Detailed Abundance Analysis}

In the majority of RGB stars it is believed that the atmosphere of the star remains an unpolluted sample of the interstellar medium out of which it was formed. This means we have small pockets of interstellar medium of different ages (enriched by different numbers of processes) conveniently covering the nuclear burning core of stars. This hot stellar core provides a useful bright background source to be absorbed in the stellar atmosphere allowing very detailed studies of the elemental abundances in these ancient gas samples. Thus, a spectroscopic analysis of the variation of the abundances of different elements seen in absorption in atmospheres of different age stars allows us to trace the detailed chemical enrichment history of a galaxy with time. Different elements are created in different circumstances (e.g., McWilliam 1997) and if we are able to determine the 

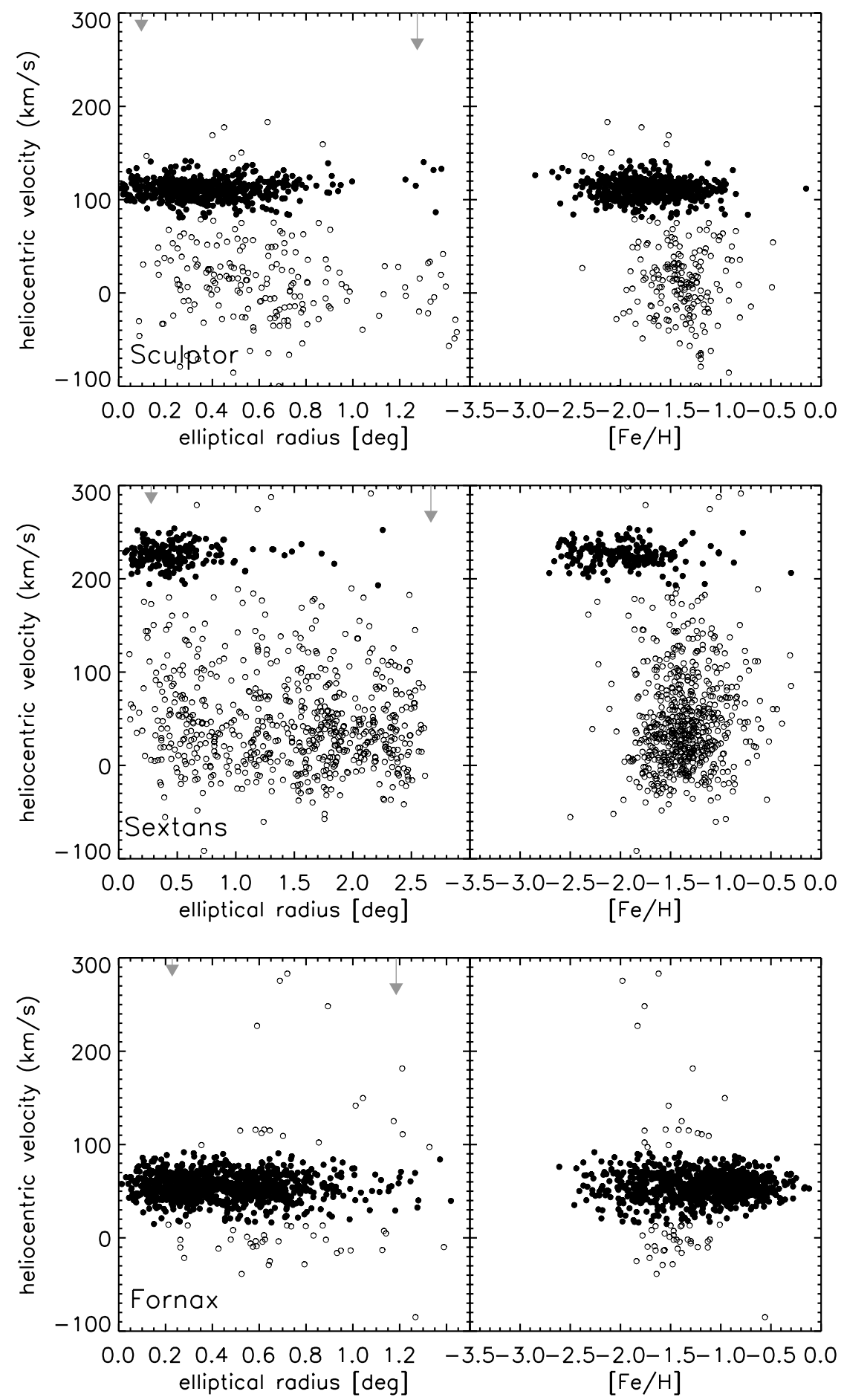

Figure 1. See legend on next page. 


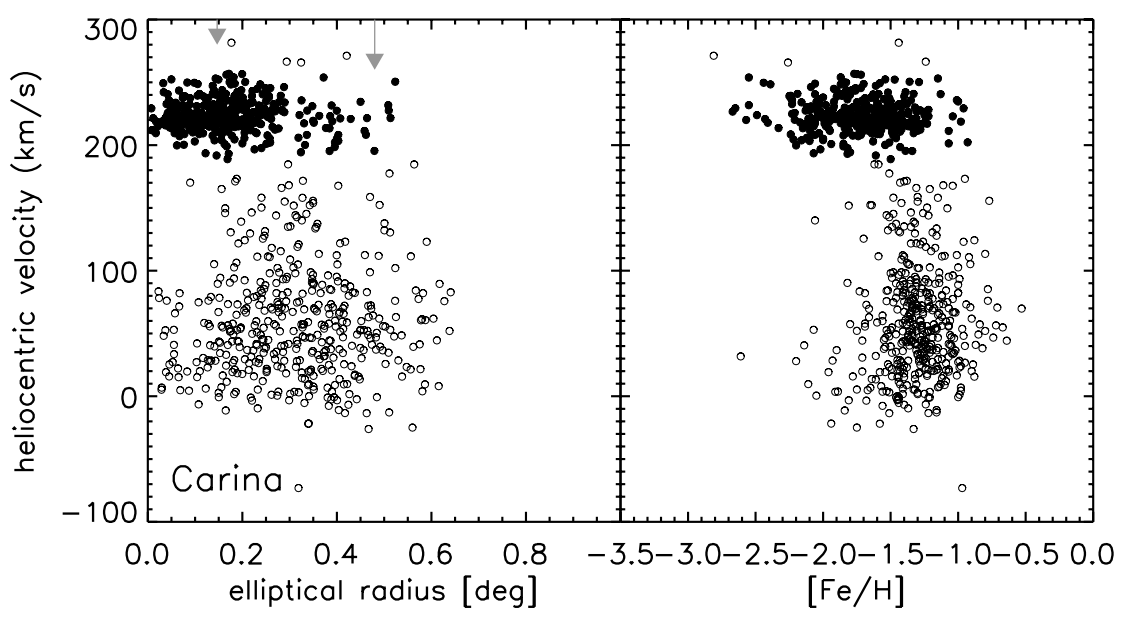

Figure 1. Velocities of stars in four dSph (solid symbols), as function of elliptical radius (left) and metallicity (right). The arrows on the left panels denote the core and tidal radius of the galaxies. Note that the foreground dwarf (open symbols) metallicities are token values; nevertheless the velocity vs. $[\mathrm{Fe} / \mathrm{H}]$ diagrams are generally a useful diagnostic of membership. From Helmi et al. (2006).

abundance of elements known to be created in a particular set of physical conditions, we can assess the importance and frequency of these conditions during the history of star formation in a galaxy. We can then compare these results between different types of galaxy, which clearly show that enrichment patterns differ strongly between galaxies, making it hard to build one type of galaxy out of another once they have formed a significant number of stars.

\section{The CaII Triplet and Kinematics}

The ideal is to be able to obtain high resolution spectra for individual stars in nearby $\mathrm{dSph}$ over a large wavelength range, and make a detailed analysis of a range of different elements along with accurate velocities. However, this is quite time consuming both in telescope time (even with VLT/FLAMES) and in analysis. One of the most simple ways to get an estimate of the metallicity of RGB stars is with the CaII triplet (e.g., Cole et al. 2004). This is a basic metallicity indicator requiring only low or intermediate spectral resolution, based on three lines around $8500 \AA$ which have been empirically calibrated from observations of stars in globular clusters stars with high resolution abundances (e.g., Armandroff \& Da Costa 1991). Assuming sufficient signal-to-noise spectra (S/N $>10$ ) it provides $[\mathrm{Fe} / \mathrm{H}]$ within typical (internal) errors of $\pm 0.1 \mathrm{dex}$, and also the radial velocity of each star with $\pm 2 \mathrm{~km} / \mathrm{s}$ accuracy. These accuracies are well suited for "quick look" surveys of the resolved stellar population of a galaxy. In the DART project these CaII triplet measurements are complementary to the high resolution observations made in the centre of each dSph. In the low resolution a much larger area is surveyed and we can assess how representative the detailed study is of the stellar population of the whole galaxy.

Within DART we have made $\mathrm{CaT}[\mathrm{Fe} / \mathrm{H}]$ determinations and direct measurements of $[\mathrm{Fe} / \mathrm{H}]$ from numerous Fe lines in high resolution spectra for same stars in the central regio of Scl and Fnx dSph (Battaglia et al. 2007, in prep). This results in useful check of the CaT calibration against direct measurements of Fe. This is the first time this has 

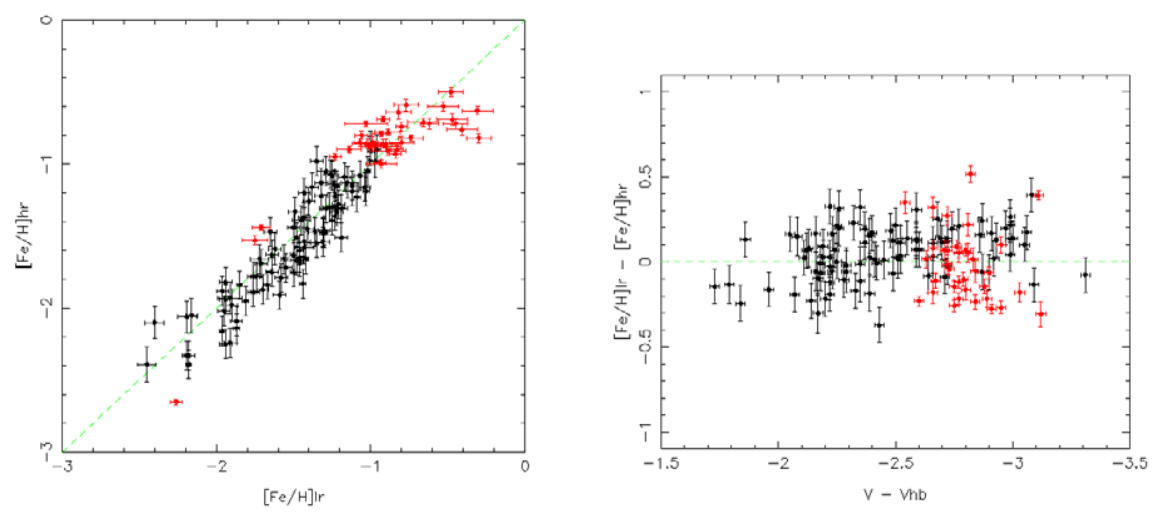

Figure 2. Comparison of the CaII triplet calibration to $[\mathrm{Fe} / \mathrm{H}]$ and direct meaurements of $[\mathrm{Fe} / \mathrm{H}]$ from high resolution spectroscopy for the same stars in the Fornax (light grey points) and Sculptor (black points) dSph galaxies. On the left-hand side are the direct meaurements, and on the right-hand side we show the difference between the two determinations of $[\mathrm{Fe} / \mathrm{H}]$ against the difference between the $\mathrm{V}$ magnitude of each star and the Horizontal Branch magnitude. From Battaglia et al. 2007, in prep.

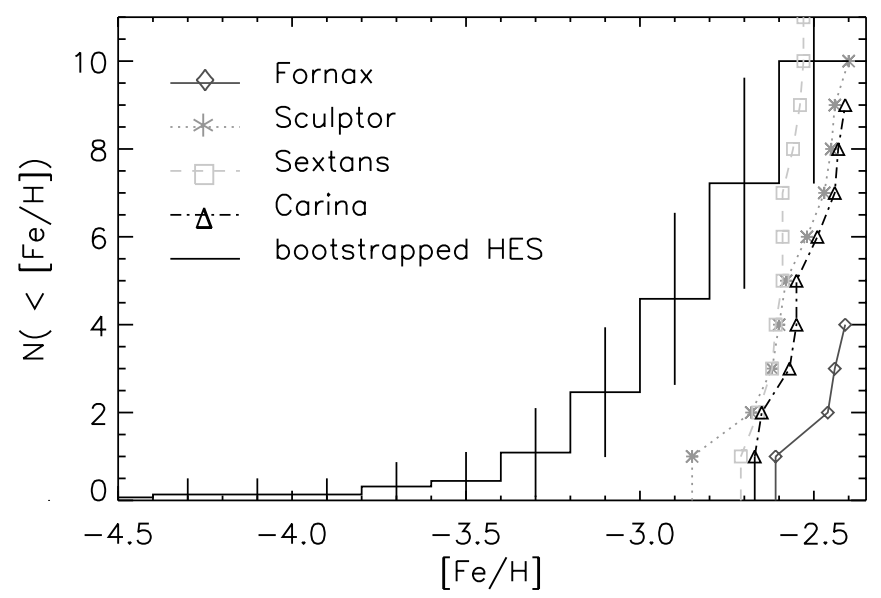

Figure 3. Comparison of the cumulative metallicity distributions of the stars in the mean bootstrapped Hamburg-ESO Survey sample and in the dSph.

been done for such a large sample of individual stars and most importantly within a galaxy instead of a globular cluster. We show the results of this comparison in Figure 2. Although there is some scatter the over-all agreement is very good.

Our first VLT/FLAMES results (Tolstoy et al. 2004), were based upon CaII triplet measurements, which clearly showed that Sculptor dSph contains two distinct stellar components with different spatial, kinematic and abundance properties. There appears to be a metal-rich, $-0.9>[\mathrm{Fe} / \mathrm{H}]>-1.7$, and a metal-poor, $-1.7>[\mathrm{Fe} / \mathrm{H}]>-2.8$ component. The metal-rich component is more centrally concentrated than the metal poor, and on average appears to have a lower velocity dispersion, $\sigma_{\text {metal-rich }}=7 \pm 1$ $\mathrm{km} / \mathrm{s}$, whereas $\sigma_{\text {metal-poor }}=11 \pm 1 \mathrm{~km} / \mathrm{s}$. A similar effect is seen in Fornax, where the metal rich stars are centrally concentrated, and the metal poor stars appear more uniformly and diffusely distributed (Battaglia et al. 2006). It is clear from the histogram of $[\mathrm{Fe} / \mathrm{H}]$ measurements that all dSph appear to lack a low metallicity tail (see Figure 3). 
The lowest metallicity star in our combined sample of more than 2000 stars for all four galaxies is $[\mathrm{Fe} / \mathrm{H}]=-2.85$. Although it is difficult to make an accurate comparison with the Galactic surveys, where the completeness can be hard to quantify, there appears to be a significantly different distribution between all the dSph and the (metal-poor) halo of the Milky Way (Christlieb et al. 2002; Helmi et al. 2006).

\section{Summary}

There are indications that the presence of two or more distinct populations is a common but not ubiquitous feature of dSph galaxies. Our preliminary analysis of Horizontal Branch stars, $v_{h e l}$ and $[\mathrm{Fe} / \mathrm{H}]$ measurements in the other galaxies in our sample (Fornax and Sextans dSph) also show similar characteristics to Sculptor, especially in the most metal poor component. Pure radial velocity studies (e.g., Wilkinson et al. 2004) have also considered the possibility that kinematically distinct components exist in Ursa Minor, Draco and Sextans dSph galaxies. Interestingly, Carina appears to go counter to this trend, and the VLT/FLAMES study finds no obvious evidence for more than one component, or even a gradient within Carina (Koch et al. 2006).

What mechanism could create two or more distinct ancient stellar components in a small dwarf spheroidal galaxy? A simple possibility is that the formation of these dSph galaxies began with an initial burst of star formation, resulting in a stellar population with a mean $[\mathrm{Fe} / \mathrm{H}] \leqslant-2$. Subsequent supernovae explosions from this initial episode could have been sufficient to cause gas (and metal) loss such that star formation was inhibited until the remaining gas could sink deeper into the centre (e.g., Mori et al. 2002). Thus the subsequent generation(s) of stars would form in a region closer to the centre of the galaxy, and have a higher average metallicity and different kinematics. Another possible cause is external influences, such as minor mergers, accretion of additional gas or the kinematic stirring by our Galaxy (e.g., Mayer et al. 2001). It might also be that events surrounding the epoch of reionisation influenced the evolution of these small galaxies (e.g., Skillman et al. 2003) and resulted in the stripping or photo-evaporation of the outer layers of gas in the $\mathrm{dSph}$, meaning that subsequent more metal enhanced star formation occurred only in the central regions.

\section{Acknowledgements}

I thank Leids Kerkhoven-Bosscha Fonds for travel support which allowed me to attend this conference.

\section{References}

Armandroff, \& Da Costa 1991 AJ, 101, 1329

Battaglia, G. \& DART 2006, A\&A, 459, 423

Belokurov, V. et al. 2006, ApJ, 647, L111

Christlieb, N. et al. 2002, Nature, 419, 904

Cole, A.A., Smecker-Hane, T.A., Tolstoy, E., Bosler, T.L. \& Gallagher, J.S. 2004 MNRAS, 347, 367

Cole, A.A., Tolstoy, E., Gallagher, J.S. \& Smecker-Hane, T.A. 2005 AJ, 129, 1465

Dolphin, A.E., Weisz, D.R., Skillman, E.D. \& Holtzman, J.A. 2005 Resolved Stellar Populations, eds. D. Valls-Gabaud \& M. Chavez (astro-ph/0506430)

Einasto, J., Saar, E., Kaasik, A. \& Chermin, A.D. 1974 Nature, 252, 111

Geisler, D., Smith, V., Wallerstein, G., Gonzalez, G. \& Charbonnel, C. 2005 AJ, 129, 1428

Helmi, A., Irwin M.J., Tolstoy, E., Battaglia G., Hill, V., et al. 2006 ApJ, 651, L121

Irwin, M. \& Hatzidimitriou, D. 1995 MNRAS, 277, 1354 
Koch, A., Grebel, E.K., Wyse, R.F.G., Kleyna, J.T., Wilkinson, M.I., Harbeck, D., Gilmore, G.F. \& Evans, N.W. (2006) AJ, 131, 1405

Lee, Y-W., Yoon, S-J., Rey, S-C. \& Chaboyer, B. 2001 Astrophysical Ages 85 Time Scales, eds. T. von Hippel, N.Mansen \& C.Simpson

Mayer, L., Governato, F., Colpi, M., Moore, B., Quinn, T., Wadsley, J., Stadel, J. \& Lake, G. 2001 ApJ, 547, L123

McWilliam, A. 1997 ARA\&A 35, 503

Mori, M., Ferrara, A. \& Madau, P. 2002 ApJ, 571, 40

Shetrone, M.D., Venn, K.A., Tolstoy, E., Primas, F., Hill, V. \& Kaufer, A. 2003 AJ, 125, 684

Skillman, E.D., Tolstoy, E., Cole, A.A., Dolphin, A.E., Saha, A., Gallagher, J.S., Dohm-Palmer, R.C. \& Mateo, M. 2003 ApJ, 596, 253

Tolstoy, E., Venn, K.A., Shetrone, M.D., Primas, F., Hill, V., Kaufer, A. \& Szeifert, T. 2003 AJ, 125, 707

Tolstoy, E., Irwin, M.J., Helmi, A., Battaglia, G., Jablonka, P., Hill, V., Venn, K.A., Shetrone, M.D., Letarte, B., Cole, A.A., Primas, F., Francois, P., Arimoto, N., Sadakane, K., Kaufer, A., Szeifert, T. \& Abel, T. 2004 ApJ, 617, 1119

Venn, K., Irwin, M., Shetrone, M.D., Tout, C.A., Hill, V. \& Tolstoy, E. 2004 AJ, 128, 1177

Wilkinson, M., Kleyna, J.T., Evans, N.W., Gilmore, G., Irwin, M.J. \& Grebel, E.K. 2004 ApJ, 611, L21

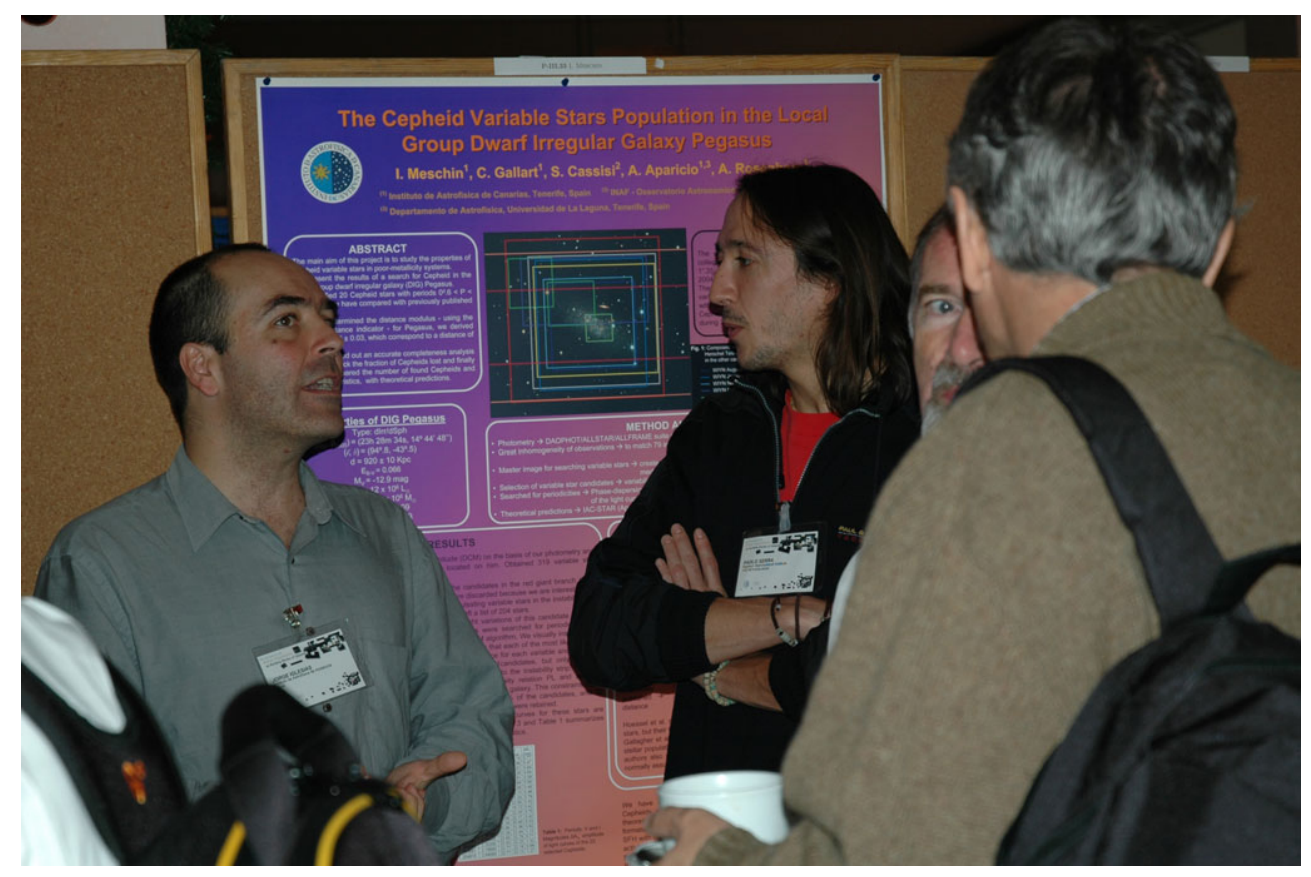

Jorge Iglesias-Páramo (left), Paolo Serra, and John Beckman (half visible). 